\title{
Preliminary results on the instrumental polarization of NIKA2-Pol at the IRAM 30m telescope
}

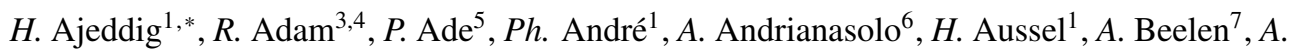

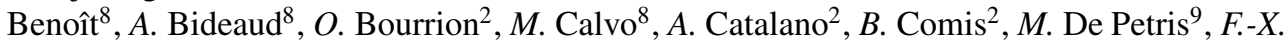
Désert $^{6}, S$. Doyle ${ }^{5}$, E. F. C. Driessen ${ }^{10}, A$. Gomez ${ }^{11}, J$. Goupy ${ }^{8}, F$. Kéruzoré ${ }^{2}, C$. Kramer ${ }^{12}, B$. Ladjelate $^{12}, G$. Lagache ${ }^{13}, S$. Leclercq ${ }^{10}, J .-F$. Lestrade ${ }^{14}, J . F$. Macías-Pérez $^{2}, A$. Maury ${ }^{1}, P$. Mauskopf $^{5,15}, F$. Mayet ${ }^{2}, A$. Monfardini ${ }^{8}, L$. Perotto ${ }^{2}, G$. Pisano ${ }^{5}, N$. Ponthieu ${ }^{6}, V$. Revéret ${ }^{1}$, $A$. Ritacco ${ }^{12}, C$. Romero ${ }^{10}, H$. Roussel ${ }^{16}, F$. Ruppin ${ }^{17}, K$. Schuster ${ }^{10}, Y$. Shimajiri ${ }^{1,18,19}, S$. $\mathrm{Shu}^{10}, A$. Sievers ${ }^{12}, C$. Tucker ${ }^{5}$, and $R$. Zylka ${ }^{10}$

${ }^{1}$ Laboratoire d'Astrophysique (AIM), CEA, CNRS, Université Paris-Saclay, Université Paris Diderot, Sorbonne Paris Cité, 91191 Gif-sur-Yvette, France

${ }^{2}$ Univ. Grenoble Alpes, CNRS, Grenoble INP, LPSC-IN2P3, 53, avenue des Martyrs, 38000 Grenoble, France

${ }^{3}$ LLR (Laboratoire Leprince-Ringuet), CNRS, École Polytechnique, Institut Polytechnique de Paris, Palaiseau, France

${ }^{4}$ Centro de Estudios de Física del Cosmos de Aragón (CEFCA), Plaza San Juan, 1, planta 2, E-44001, Teruel, Spain

${ }^{5}$ Astronomy Instrumentation Group, University of Cardiff, UK

${ }^{6}$ Univ. Grenoble Alpes, CNRS, IPAG, 38000 Grenoble, France

${ }^{7}$ Institut d'Astrophysique Spatiale (IAS), CNRS and Université Paris Sud, Orsay, France

${ }^{8}$ Institut Néel, CNRS and Université Grenoble Alpes, France

${ }^{9}$ Dipartimento di Fisica, Sapienza Università di Roma, Piazzale Aldo Moro 5, I-00185 Roma, Italy

${ }^{10}$ Institut de RadioAstronomie Millimétrique (IRAM), Grenoble, France

${ }^{11}$ Centro de Astrobiología (CSIC-INTA), Torrejón de Ardoz, 28850 Madrid, Spain

${ }^{12}$ Instituto de Radioastronomía Milimétrica (IRAM), Granada, Spain

${ }^{13}$ Aix Marseille Univ, CNRS, CNES, LAM (Laboratoire d'Astrophysique de Marseille), Marseille, France

${ }^{14}$ LERMA, Observatoire de Paris, PSL Research University, CNRS, Sorbonne Universités, UPMC Univ. Paris 06, 75014 Paris, France

${ }^{15}$ School of Earth and Space Exploration and Department of Physics, Arizona State University, Tempe, AZ 85287

${ }^{16}$ Institut d'Astrophysique de Paris, CNRS (UMR7095), 98 bis boulevard Arago, 75014 Paris, France

${ }^{17}$ Kavli Institute for Astrophysics and Space Research, Massachusetts Institute of Technology, Cambridge, MA 02139, USA

${ }^{18}$ National Astronomical Observatory of Japan, Osawa 2-21-1, Mitaka, Tokyo 181-8588, Japan

${ }^{19}$ Department of Physics and Astronomy, Graduate School of Science and Engineering, Kagoshima University, 1-21-35 Korimoto, Kagoshima, Kagoshima 890-0065, Japan

Abstract. Clarifying the role of magnetic fields in the star formation process is crucial. Observations have already shown that magnetic fields play an important role in the early stages of star formation. The high spatial resolution $(\sim 0.01$ to $0.05 \mathrm{pc}$ ) provided by NIKA2-Pol $1.2 \mathrm{~mm}$ imaging polarimetry of nearby clouds will help us clarify the geometry of the B-field within dense cores and molecular

\footnotetext{
*e-mail: hamza.ajeddig@cea.fr
} 
filaments as part of the IRAM 30m large program B-FUN. There are numerous challenging issues in the validation of NIKA2-Pol such as the calibration of instrumental polarization. The commissioning phase of NIKA2-Pol is underway and is helping us characterize the intensity-to-polarization "leakage" pattern of the instrument. We present a preliminary analysis of the leakage pattern and its dependence with elevation. We also present the current leakage correction made possible by the NIKA2 pipeline in polarization mode based on the NIKA2-Pol commissioning data taken in December 2018. Based on reduced Stokes I, Q, U data we find that the leakage pattern of NIKA2-Pol depends on elevation and is sensitive to the focus of the telescope.

\section{Introduction}

Herschel observations of nearby molecular clouds support a filament paradigm for star formation in which molecular filaments represent a key evolutionary step [2, 3, 8, 9]. The Herschel data indicate that filaments have a common inner width of $\sim 0.1 \mathrm{pc}[5,6]$. In parallel, dust polarization observations of the whole sky with the Planck satellite have revealed a regular morphology for magnetic field lines on large scales in interstellar clouds; the orientation of the field tends to be parallel to low-density structures and perpendicular to high-density filaments [11]. This suggests that the magnetic field plays a crucial role in the growth of ISM structure leading to star formation. The transition between the two regimes (with a B-field mostly parallel and mostly perpendicular to the orientation of structures, respectively) is still poorly understood. Clarifying the nature of this transition requires higher spatial resolution than Planck and a detailed statistical analysis. The angular resolution of Planck polarization data ${ }^{1}$ is also insufficient to investigate the link between the large-scale $(>>0.1 \mathrm{pc})$ magnetic field in molecular clouds and the small-scale $(<<0.1 \mathrm{pc})$ magnetic field relevant to the formation of individual protostars in dense cores.

NIKA2-Pol is the polarization channel of NIKA2, the new millimeter continuum camera on the IRAM 30m telescope [1]. NIKA2-Pol will soon provide $1.2 \mathrm{~mm}$ polarimetric imaging of a selected sample of star-forming molecular filaments at 12 arcsec resolution (or $\approx 8 \mathrm{mpc}$ in the nearest clouds at $d \sim 140 \mathrm{pc}$ ), as part of the large program B-FUN ${ }^{2}$ (see also [4] for the scientific motivation). When observing in polarization mode, numerous effects coming from the polarimeter itself or the telescope influence the polarization measurements, which need to be characterized before starting science observations. In particular, instrumental polarization, i.e., the conversion of a fraction of unpolarized incident signal into polarized emission, here expressed in the form of an intensity-to-polarization leakage pattern (cf. Fig. 1), needs to be carefully calibrated as part of the polarimeter's commissioning. The NIKA2-Pol polarimeter, currently in the commissioning stage ${ }^{3}$, benefits from previous experience of polarization measurements with the NIKA1 pathfinder, which tested and demonstrated the feasibility of millimeter continumm polarimetry with KIDs and a fast $(3 \mathrm{~Hz})$ and continuously rotating half-wave plate [12]. The NIKA2 instrument was itself tested in polarization mode during the week of December 5-11, 2018 at Pico Veleta. As part of current efforts to characterize the performance of NIKA2-Pol and its ability to deliver reliable polarization maps, the present paper gives an overview of the instrumental polarization affecting the data.

\footnotetext{
${ }^{1} \sim 10$ arcmin or $\sim 0.4 \mathrm{pc}$ in the nearest star-forming clouds at $d \sim 140 \mathrm{pc}$

${ }^{2}$ http://www.iram.es/IRAMES/mainWiki/Continuum/NIKA2/Main

${ }^{3}$ https://lpsc-indico.in2p3.fr/Indico/event/1765/speakers
} 


\section{Instrumental polarization: "leakage” pattern}

During the Dec. 2018 commissioning week, we repeatedly observed the planet Uranus, an unpolarized source (see [14], where a polarization fraction of $0.10 \pm 0.04 \%$ is reported for Uranus), in order to quantify the instrumental polarization affecting NIKA2-Pol data. We analyzed the resulting data in Stokes I, Q, U obtained at different elevations and focus positions. Qualitatively, the leakage pattern observed with NIKA2-Pol has the shape of a cloverleaf consisting of a central positive lobe surrounded by a negative quadrupolar pattern (see Fig. 1). The reason for this pattern is still under investigation. To first order at least, the leakage pattern in Stokes U can be obtained by rotating the leakage pattern in Stokes Q by $\sim 45^{\circ}$. As part of the NIKA2-Pol commissioning run, we performed several observations of Uranus to determine if/how the leakage pattern changes with elevation (due to the system, e.g. telescope, optical path, or polarimeter) and/or whether it changes with the focus position. Instrumental polarization affecting other millimeter and submillimeter polarimeters, such as POL-2/SCUBA-2 on the James Clerk Maxwell Telescope (JCMT) [7], POLKA/LABOCA at the APEX telescope [14], or XPOL at the IRAM 30m telescope [13], has been characterized following a similar approach (observing an unpolarized source such as Uranus).

\subsection{Dependence of the leakage pattern on elevation}

The Uranus maps taken at low and high elevations show that the leakage pattern varies with elevation, and suggest that the level of instrumental polarization is higher at low elevation. As an illustration, Fig. 1 shows Stokes I, Q and U maps obtained for Uranus at two elevations, $\sim 52^{\circ}$ (top panel) and $31^{\circ}$ (bottom panel). As can be seen in Fig. 1, the maximum fractional instrumental polarization varies from $0.45 \%$ to $1.84 \%$ in $\left|U / I_{\text {peak }}\right|$ and from $1.27 \%$ to $2.16 \%$ in $\left|Q / I_{\text {peak }}\right|$ when source elevation changes from $52^{\circ}$ to $31^{\circ}$, while the leakage pattern conserves the same cloverleaf shape. Due to the lack of good Uranus data at closely spaced elevations, the detailed manner in which the instrumental polarization level varies with elevation remains very uncertain, especially in the critical elevation range between $\sim 20^{\circ}$ and $\sim$ $45^{\circ}$ at which many potential scientific targets would be observed.

\subsection{Dependence of the leakage pattern on focus}

We found that the leakage pattern changed significantly even for small $( \pm 0.3 \mathrm{~mm})$ differences in the focus position of the telescope (see Fig. 2). We tried to assess how much the leakage pattern would depart from the nominal pattern (for well-focused observations) in the case of observations taken while the telescope is (slightly) out of focus. Figure 2 shows a schematic diagram summarizing the typical evolution of the leakage pattern as a function of offset from the good focus position. This was estimated from a series of Uranus defocused maps. We found that the maximum fractional amplitude of the leakage or instrumental polarization pattern $\left(\left|\mathrm{Q} / \mathrm{I}_{\text {peak }}\right|_{\max }\right.$ and $\left.\left|\mathrm{U} / \mathrm{I}_{\text {peak }}\right|_{\max }\right)$ increases by only $\sim 0.2 \%$ as long as the focus position is off by less than $\pm 0.3 \mathrm{~mm}$ from the optimum focus correction (along $\mathrm{Z}$ ). However, the shape of the leakage pattern changes quite significantly (and even loses its cloverleaf shape) at even small focus offsets of $\pm 0.3 \mathrm{~mm}$. To further quantify the dependence of the leakage pattern with focus, a larger number of defocused maps of Uranus taken at smaller focus offsets (e.g. $\pm 0.1 \mathrm{~mm}, \pm 0.15 \mathrm{~mm}, \pm 0.2 \mathrm{~mm}, \pm 0.25 \mathrm{~mm}$ ) from the optimal focus value will have to be observed. This will be one of the main purposes of the next NIKA2-Pol commissioning run. 

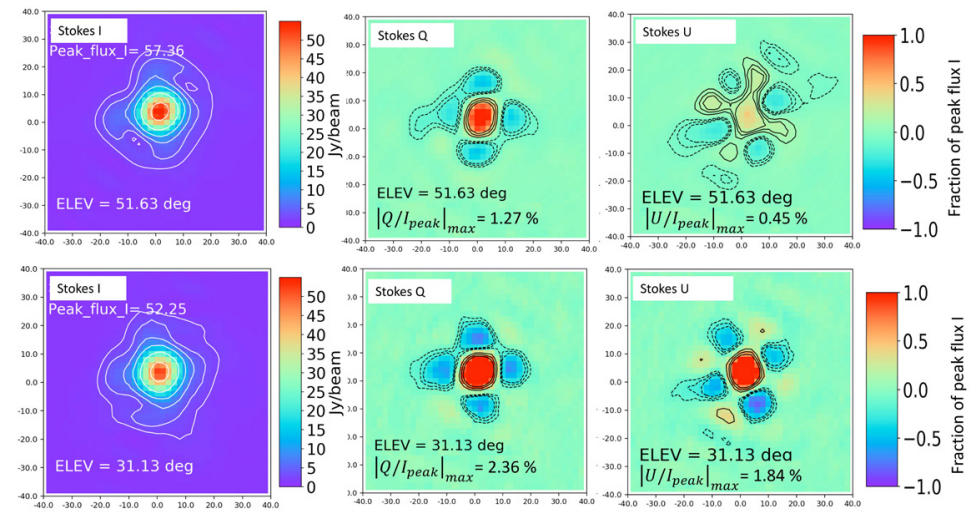

Figure 1. Examples of Stokes (I, Q, U) maps obtained for Uranus (in Nasmyth coordinates). Uranus being an unpolarized source, the signals detected in Stokes Q, U correspond to instrumental polarization, which should be corrected for as part of the reduction of NIKA2 polarization data (a.k.a leakage correction). The color bar shows the fraction of $\mathrm{U} / \mathrm{I}_{\text {peak }}$ or $\mathrm{Q} / \mathrm{I}_{\text {peak }}$. The leakage patterns in both Stokes $\mathrm{Q}$ and $\mathrm{U}$ vary from high to low elevation with a more deformed pattern in Stokes $\mathrm{U}$. The level of instrumental polarization (expressed as a fraction $\mathrm{U} / \mathrm{I}_{\text {peak }}$ or $\mathrm{Q} / \mathrm{I}_{\text {peak }}$ ) increases from $0.45 \%$ to $1.84 \%$ in Stokes U (right column) and from $1.27 \%$ to $2.36 \%$ in Stokes Q (middle column) when source elevation decreases from $52^{\circ}$ to $31^{\circ}$.

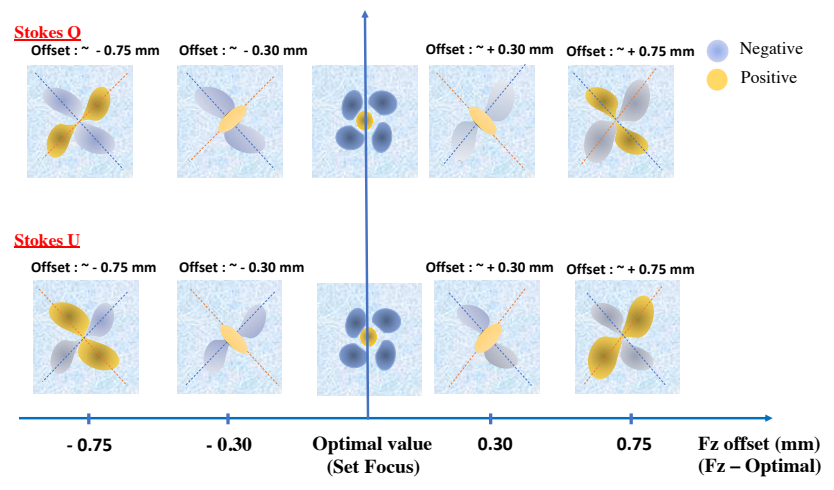

Figure 2. Schematic diagram in Nasmyth coordinates summarizing how the instrumental polarization pattern (Stokes $\mathrm{Q}$ and $\mathrm{U}$ emission maps from an unpolarized source) varies as a function of the offset from the optimum focus position (along the $\mathrm{Z}$ axis). The typical cloverleaf pattern seen at the best focus position becomes more severely distorted as the applied focus correction is farther away from its optimum value.

\section{Correcting for the leakage pattern}

We used the best Stokes Q, U maps of Uranus taken during the Dec. 2018 commissioning week to verify that a leakage correction procedure similar to that devised by Ritacco et al. [12] 
for NIKA1 was properly implemented in the NIKA2 IDL pipeline (still under development). First, we applied the leakage correction directly to the Uranus map used to estimate the leakage pattern (i.e., observed at $52^{\circ}$ elevation, shown in the top row of Fig. 1). The residual Stokes $\mathrm{Q} / \mathrm{I}_{\text {peak }}$ and $\mathrm{U} / \mathrm{I}_{\text {peak }}$ maps obtained after correcting the data for the leakage pattern are displayed in Fig. 3f and Fig. 31, respectively. These residual $\left|Q / I_{\text {peak }}\right|$ and $\left|U / I_{\text {peak }}\right|$ maps are less than $0.1 \%$ in amplitude, suggesting that the leakage-correction procedure has been properly implemented in the NIKA2 pipeline. Figure 3 also shows the residual Stokes $Q / I_{\text {peak }}$, $\mathrm{U} / \mathrm{I}_{\text {peak }}$ maps obtained when applying the leakage-correction procedure to Uranus maps taken at other elevations than the map used to estimate the leakage pattern. We found that for maps taken at elevations $>45^{\circ}$ the magnitude of the residuals is lower than $0.3 \%$ (in $\left|\mathrm{Q} / \mathrm{I}_{\text {peak }}\right|$ ) and $0.5 \%$ (in $\left.\left|\mathrm{U} / \mathrm{I}_{\text {peak }}\right|\right)$ after leakage correction, but for maps at low elevations $\left(\sim 20^{\circ}-30^{\circ}\right)$ the residuals become significantly higher, up to $0.8 \%$ in $\left|\mathrm{Q} / \mathrm{I}_{\text {peak }}\right|$ and $2 \%$ in $\left|\mathrm{U} / \mathrm{I}_{\text {peak }}\right|$ (Fig. 3). Similar correction tests will be investigated with polarization residuals obtained at other elevations $\left(\mathrm{e} . \mathrm{g} \sim 31^{\circ}\right.$ ) to test this hypothesis. According to these preliminary results, it seems
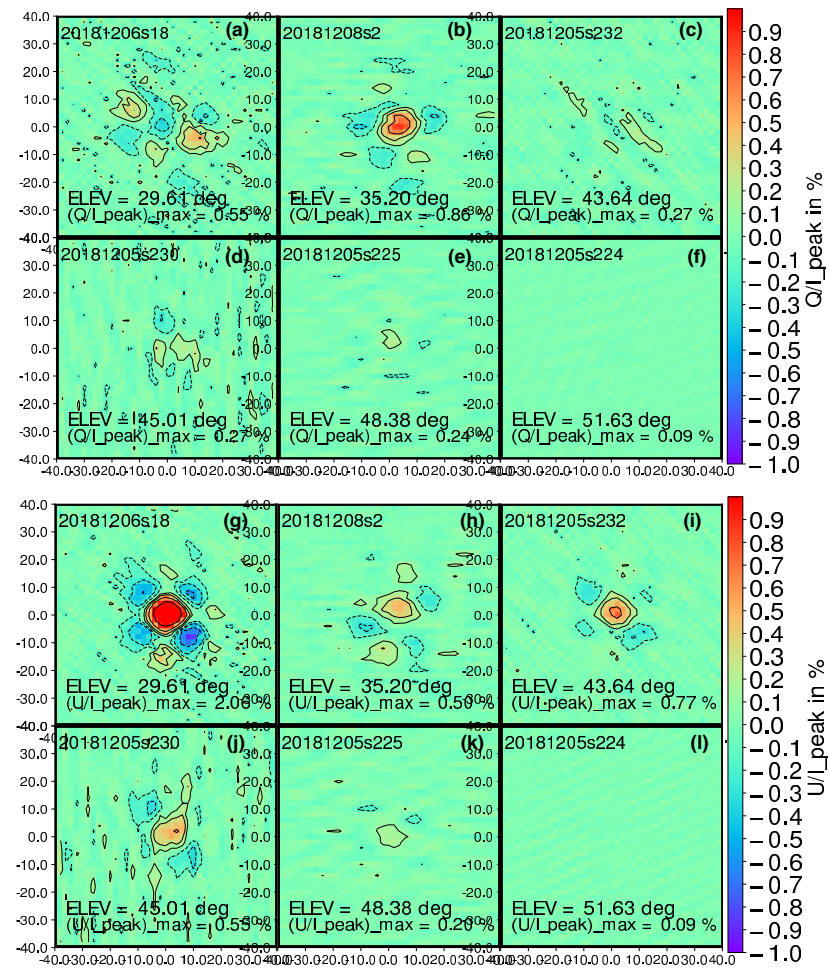

Figure 3. Residual Stokes $\mathrm{Q} / \mathrm{I}_{\text {peak }}$ and $\mathrm{U} / \mathrm{I}_{\text {peak }}$ maps (expressed in \% of $\mathrm{I}_{\text {peak }}$ ) obtained after correcting each of the individual Uranus maps, taken at different elevations, by a single leakage pattern (from Uranus map taken at $52 \mathrm{deg}$ elevation, (f) and (l) panels). The contours show levels of $-2 \%,-1 \%$, $-0.5 \%,-0.2 \%,+0.2 \%,+0.5 \%,+1.0 \%,+2.0 \%$. Note how the maps taken at elevations different from the one used to correct for the leakage show non-negligible residuals (up to $\sim 2 \%$ ).

that a proper correction of NIKA2-Pol observations for instrumental polarization effects will require a good characterization of the leakage pattern at an elevation close to that of the observed data $\left(\sim \pm 5^{\circ}\right)$. 


\section{Conclusions}

NIKA2-Pol will peer into the magnetic field of star-forming filaments as part of the IRAM $30 \mathrm{~m}$ large program B-FUN. The instrumental polarization of NIKA2-Pol will be better characterized in forthcoming commissioning runs. The current NIKA2-Pol pipeline includes a method to correct for instrumental polarization which was already validated as shown in this paper. One of the next commissioning steps will be to obtain good maps of Uranus in polarization mode at closely-spaced elevations and for a fine grid of focus settings. Determining how the intensity-to-polarization leakage pattern varies with elevation and focus in more detail will allow us to use our correction method on NIKA2-Pol scientific data.

\section{Acknowledgements}

We would like to thank the IRAM staff for their support during the campaigns. The NIKA dilution cryostat has been designed and built at the Institut Néel. In particular, we acknowledge the crucial contribution of the Cryogenics Group, and in particular Gregory Garde, Henri Rodenas, Jean Paul Leggeri, Philippe Camus. This work has been partially funded by the Foundation Nanoscience Grenoble and the LabEx FOCUS ANR-11-LABX-0013. This work is supported by the French National Research Agency under the contracts "MKIDS", "NIKA" and ANR-15-CE31-0017 and in the framework of the "Investissements d'avenir" program (ANR-15-IDEX-02). This work has benefited from the support of the European Research Council Advanced Grant ORISTARS under the European Union's Seventh Framework Programme (Grant Agreement no. 291294). We acknowledge fundings from the ENIGMASS French LabEx (R. A. and F. R.), the CNES post-doctoral fellowship program (R. A.), the CNES doctoral fellowship program (A. R.) and the FOCUS French LabEx doctoral fellowship program (A. R.). R.A. acknowledges support from Spanish Ministerio de Economía and Competitividad (MINECO) through grant number AYA2015-66211-C2-2.

\section{References}

[1] R. Adam et al., Astron. Astrophys. 609, A115 (2018)

[2] Ph. André et al., Astron. Astrophys. 518, L102 (2010)

[3] Ph. André et al., in Protostars and Planets VI, 27-51 (2014)

[4] Ph. André et al., PASA. 36, e029 (2019)

[5] D. Arzoumanian et al., Astron. Astrophys. 529, L6 (2011)

[6] D. Arzoumanian et al., Astron. Astrophys. 621, A42 (2019)

[7] P. Friberg et al., Millimeter, Submillimeter, and Far-Infrared Detectors and Instrumentation for Astronomy IX 10708 3M (2018)

[8] V. Könyves et al., Astron. Astrophys. 584, A91 (2015)

[9] P. Palmeirim et al., Astron. Astrophys. 550, A38 (2013)

[10] L. Perotto et al., submitted to Astron. Astrophys.

[11] Planck collaboration et al., Astron. Astrophys. 594, A1 (2016)

[12] A. Ritacco et al., Astron. Astrophys. 599, A34 (2017)

[13] C. Thum et al., PASP. 120, 777-790 (2008)

[14] H. Wiesemeyer et al., PASP. 126, 1027 (2014) 